\title{
Sonoporation - One of the promising techniques to treat oral cancer
}

Diksha Singh ${ }^{1}$, Anand S Tegginamani ${ }^{2}$, Manish Kumar Singh ${ }^{3}$, Parikshit Sharma ${ }^{4}$

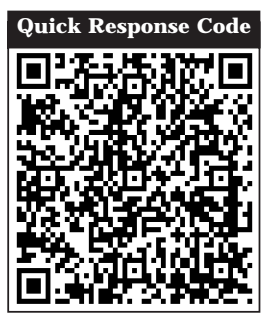

doi: $10.5866 / 2014.631608$

${ }^{1}$ Assistant Professor

${ }^{4}$ Senior resident

Oral Pathology FODS,

King George's Medical University, Lucknow, India

${ }^{2}$ Associate Professor

Department of Oral pathology and Microbiology,

Hitkarni Dental College, J abal pur, Madhya Pradesh,

${ }^{3}$ Assistant professor

Department of Community Medicine,

BRD Medical college ,Gorakhpur, Uttar Pradesh

\section{Article Info:}

Received: April 12, 2014

Review Completed: May 11, 2014

Accepted: J une 10, 2014

Available Online: October, 2014 (www.nacd.in)

(C) NAD, 2014 - All rights reserved

\section{Email for correspondence:}

diksh18@yahoo.com

\begin{abstract}
:
Recent studies demonstrate that ultrasound can be used to deliver compounds into viable cells advantageous possibilities in the field of precancer and cancer management. The delivery is facilitated through ultrasound waves i.e. sonoporation, principle is the formation of temporary pores in the cell membrane induced by ultrasound. Our review focuses on the study of sonoporation mechanisms in order to achieve optimal delivery outcome such as high delivery efficiency and minimal cell death. Ultrasound increased the transmembrane current as a direct result of decreased membrane resistance due to pore formation. The ability to real time monitor sonoporation of cells provides a novel and necessary tool to study the dynamic sonoporation process and obtain optimal delivery parameters. We reviewed the literature so that we can make a clear sense to utilize the sonoporation in the field of cancer and precancer lesions of oral cavity.
\end{abstract}

Key words: Sonoporation, Ultrasound, Cavitation, Cancer And Precancer.

\section{Introduction}

The ultrasound exposure generates bio-effects resulting in tissue heating, shear stress, and cavitation, which have been exploited for therapeutic applications. Ultrasound cavitation, enhanced by injected micro bubbles, perturbs cell membrane structures to cause sonoporation and increases the permeability to bioactive materials. Ultrasoundmediated gene delivery has been applied to heart, blood vessel, lung, kidney, muscle, head and neck tumour with enhanced genetransfection efficiency. ${ }^{1}$

\section{Definition of Sonoporation}

Sonoporation is defined as the interaction of ultrasound with ultrasonic contrast agents to temporarily permeabilize the cell membrane allowing for the uptake of various substances such as DNA, drugs, and other therapeutic compounds, from the extracellular environment. Sonoporation 
is used in the delivery of therapeutic agents including genetic material, proteins and chemotherapeutic agents into the cell, in a cell disruption process called transfection or transformation. It employs the acoustic cavitation of microbubbles to enhance delivery of these large molecules. This technique is similar to, and in some cases found superior to, electroporation. ${ }^{2}$

\section{Mechanism of Action}

The technique of sonoporation is based on ultrasonic waves. The source of these waves is a piezoelectric crystal transducer made of leadzirconate-titanium or barium titanate. These waves are produced in response to an electrical impulse in the piezoelectric crystal, allowing the conversion of electrical energy into mechanical or vibrational energy. It has been hypothesized that ultrasound energy, often amplified by use of microbubble activities, generates transient, non-specific pores on cell membranes, a process termed sonoporation. The transient pores allow a limited time window for the otherwise non-permeable extracellular agents to enter the cells. ${ }^{2}$

\section{Advantages}

- Safe and efficient intracellular delivery of drugs and genes is critically important in targeted cancer treatment and gene therapy application.

The confirmation of the delivery of compound into cells was made by using markers such as plasmid GFP. ${ }^{3}$ Their study focused on the study of sonoporation mechanisms in order to achieve optimal delivery outcome such as high delivery efficiency and minimal cell death.

- Studies have demonstrated that ultrasonication techniques are able to deliver DNA, proteins, and other formulations of molecules into the viable cells.

According to study conducted by Yamatomo et al (2013) Boron neutron capture therapy (BNCT) is a selective radiotherapy that is dependent on the accumulation of 10B compound in tumors. Low-intensity ultrasound produces a transient pore on cell membranes, sonoporation, which enabled extracellular materials to enter cells. ${ }^{4}$

\section{Effect of Cavitation}

The cavitation is the formation of gaseous cavities in medium due to pressure variation in the medium caused by ultrasound waves. This involves both rapid growth and collapse of a bubble (inertial cavitation), or theslow oscillatory motion of a bubble in a medium (stable cavitation). Collapse of cavitation bubbles releases a shock wave that can causestructural alteration in the surrounding tissue leads to the disordering of the lipid bilayers and formation of aqueous channels in the skin, allowing entry of extracellular agents into the cytoplasm. Even though cavitation processes are well known to be stochastic in nature, with the further complication of encapsulated UCAs, intrinsic variations in microbubble shell uniformity may also be a factor, especially for the process of sonic cracking. ${ }^{5}$

\section{Review of literature}

Some authors found that an administration of cdtB-expressing plasmid followed by sonoporation induced marked growth inhibition of Ca9-22 cells and apoptotic cells were also observed in vitro and in vivo. These findings suggested that local administration of cytotoxic agents with sonoporation is a useful method for molecular cancer therapy. The patient will be saved from adverse effects of anticancer drugs which usually act as double edged swords. ${ }^{2,4}$

The confirmation of the delivery of compound into cells was made by using markers such as plasmid GFP. Some research focused on the study of sonoporation mechanisms in order to achieve optimal delivery outcome such as high delivery efficiency and minimal cell death.

Apoptosis is an organized process of cell death, which occurs naturally for unneeded cells. This process may be used in future for killing cancerous cells and other cells of benign growths before malignant change or size reduction of the growth before surgeries. Ashush et al (2000) and Ando et al (2006), concluded that exposure of cells to ultrasonic cavitation was shown to induce apoptosis in addition to the conventionally reported instantaneous cell lysis and necrotic disintegration. ${ }^{5-7}$ 
Stimulatory effect of dentin bone morphogenic protein was more than bone bone morphogenic protein for reparative dentinogenesis. ${ }^{8}$ Bone morphogenetic proteins (BMPs) are members of the TGF-â superfamily and play critical role in skeletal development, bone formation and stem cell differentiation.

Ashush et al. (2000) and Ando et al (2006), concluded that exposure of cells to ultrasonic cavitation was shown to induce apoptosis in addition to the conventionally reported instantaneous cell Iysis and necrotic disintegration. ${ }^{9}$

Additional therapy guided with sonoporation dramatically inhibited primary tumour growth throughout the course of the disease, with median survival increases of up to $10 \%$ demonstrated in adeno-carcinomas of pancreatic xenografts in comparison to the control groups. ${ }^{10}$

In the study conducted by I wanaga et al, in vitro delivery and in vivo transfection systems for BLM and the cdtB geneinto oral squamous cell carcinomas using sonoporation was established.This process may be used in future for killing cancerous cells and other cells of benign growths before malignant change or size reduction of the growth before surgeries

\section{Applications of Sonoporation ${ }^{8}$}

- Gene delivery

- $\quad$ Osteoinduction ${ }^{9}$

- Induction of dental pulp stem cell differentiation into odontoblasts

- $\quad$ Site specific gene delivery

- DNA transfer

- Local Drug delivery

- Targeted Drug delivery

- Tumor cell killing

- Induction of Apoptosis

Principle of gene therapy ${ }^{11}$

1. An abnormal gene could be swapped for a normal gene through homologous recombination.
2. The abnormal gene could be repaired through selective re-verse mutation, which returns the gene to its normal function.

3. The regulation (the degree to which a gene is turned on or off) of a particular gene could be altered.

\section{Physical Methods to Enhance Gene Delivery ${ }^{12}$}

1. Electroporation: Electroporation is a method that uses short pulses of high voltage to carry DNA across the cell membrane. This shock is thought to cause temporary formation of pores in the cell membrane, allowing DNA molecules to pass through. Electroporation is generally efficient and works across a broad range of cell types. However, a high rate of cell death follow-ing electroporation has limited its use, including clinical appli-cations.

2. Gene Gun: The use of particle bombardment, or the gene gun, is another physical method of DNA transfection. In this technique, DNA is coated with gold particles and loaded into a device which generates a forceto achieve penetration of DNA/gold into the cells.eg:- If the DNA is integrated in the wrong place in the genome, for example in a tumor suppressor gene, it could induce a tumor. This has occurred in clinical trials for X-linked severe combined immunodeficiency (X-SCID) patients, in which hematopoietic stem cells were transduced with a corrective transgene using a retrovirus, and this led to the development of $\mathrm{T}$ cell leukemia in 3 of 20 patients.

3. Sonoporation: Sonoporation uses ultrasonic frequencies to deliver DNA into cells. The process of acoustic cavitation is thought to disrupt the cell membrane and allow DNA to move into cells.

4. Magnetofection: In a method termed magnetofection, DNA is complexed to a magnetic particles, and a magnet is placed underneath the tissue culture dish to bring DNA complexes into contact with a cell monolayer.

\section{Other Application in Dentistry}

Sonoporation therapy, a promising tool for locally enhancing chemotherapeutic drug delivery, with the potential to augment cancer treatments for many primary malignancies. The treatment of retinoblastoma has been increasingly focused on 
localizing therapy to the eye. This approach has the potential advantage of allowing the eye to beinjured by preserving some level of functional vision while minimizing systemic side effects. Thus, sonoporation will prove to bea valuable adjuvant to chemotherapy in retinoblastoma, where treatment is often limited by drug delivery. ${ }^{13}$

An important factor for the success of clinical therapy treatment for squamous cell carcinoma is the state of lymph node metastasis. In the maxilla, squamous cell carcinomas often invade into the maxillary sinus, whereas in the mandible, extension into the floor of the mouth often occurs. Although clinical treatment is generally accomplished with surgery in those cases, the prognosis is not particularly good and the use of antiproliferative agents, such as 5-fluorouracil, mitomycin $C$ and $B L M$, has been reported to remarkably improve the therapeutic effect. ${ }^{4}$ Sonoporation efficiency is also related to the microbubble concentration and composition, as well as to the microbubble to cell ratio. ${ }^{5,14,15}$

Additional therapy guided with sonoporation dramatically inhibited primary tumour growth throughout the course of the disease, with median survival increases of up to $10 \%$ demonstrated in adenocarcinomas of pancreatic xenografts in comparison to the control groups. ${ }^{16}$

The sonoporation mediated gene transfer technique was also evaluated for transfection efficiency and safety for gene delivery in comparison with other methods. The results in all the similar studies concluded that sonoporation gene therapy might be the safest technique to be used in actual clinical practice. ${ }^{17,18}$

\section{F uture Challenges of sonoporation}

One of the challenges of sonoporation is to develop methods to control the induced bioeffects, in particular reversible and irreversible sonoporation. Sonoporation has complex dosimetry because many parameters can be identified. The efficiency of sonoporation can influence Cavitation activity via US output parameters (the term originally used by Hallow et al 9), ie, by varying the US peak negative pressure, exposure duration, frequency, duty cycle, and pulse length. ${ }^{14}$
Sonoporation efficiency is also related to the microbubble concentration and composition, as well as to the microbubble to cell ratio. 5,19,20

According to Paul et al selective introduction of therapeutic agents to the interior of cells remains a challenging problem, principally because the lipid membrane acts as an impermeable barrier to most water-soluble molecules. To address this issue, physical approaches are attractive as they typically offer more generic applicability compared with viral or biochemical counterparts. ${ }^{3}$ Of the approaches devised thus far, both electroporation and photoporation have demonstrated impressive in vitro results. However, their development for use in general (non-topical) clinical settings would typically require an invasive procedure. In contrast, using ultrasound to mediate molecular delivery to remote anatomical sites has exciting potentials it may be applied extra-corporeally, and may also be focused to specific localized regions internally. ${ }^{20}$

\section{Future advancements}

Together with the progression of nanoscaledrug delivery systems, advances in nanoscale imaging suggest the potential for the development of multifunctional "smart" nanoparticles that may facilitate the realization of individualized cancer therapy. Almost all types of nanoparticles including polymeric nanoparticles, nanocrystals, polymeric micelles, dendrimers, and carbon nanotubes have been evaluated for their suitability as multifunctional nanoparticles that can be applied for simultaneous in vivo imaging and treatment of cancers. ${ }^{20}$

\section{Conclusion}

Thus, its simplicity and non invasiveness may provide a new avenue for microinjecting various substances into a wide range of living tissues. The efficiency of sonoporation can influence cavitation activity via US output parameters (the term originally used by Hallow et al), ie, by varying the US peak negative pressure, exposure duration, frequency, duty cycle, and pulse length. Sonoporation efficiency is also related to the microbubble concentration and composition, as well as to the microbubble to cell ratio. With this we can envision a whole gamut of newer technologies and products in the foreseeable future dentistry. ${ }^{13,20}$ 


\section{References}

1. I wanaga $\mathrm{K}$, Tominaga $\mathrm{K}$, Yamamoto K, Habu M, Maeda $\mathrm{H}$, Akifusa $S$ et al. Local delivery system of cytotoxic agents to tumors by focused sonoporation: Cancer GeneTherapy 2007; 14:354-363.

2. Koneru et al. Therapeutic ultrasound. J ournal of Orofacial Sciences 2012; 4(1):3-6.

3. Pan H, Zhou Y, Sieling F, Shi J , Cui J , Deng C. Sonoporation of cells for drug and gene delivery. Conf Proc IEEE Eng Med Biol Soc 2004; 5:3531-3534.

4. J ing Ma1, Lian Fang Du, Ming Chen, Hang Hui Wang, Ling Xi Xing. Drugloaded nanomicrocapsules delivery system mediated by ultrasoundtargeted microbubble destruction: A promising therapy method. Biomedical Reports 2013; 1:506-510.

5. Paul Prentice, Alfred Cuschieri, Kishan Dholakia, Mark Prausnitz, Paul Campbell. Membrane disruption by optically controlled microbubble cavitation. Nature Physics 2005;1:107-110.

6. Soheyl Sheikh, Shambulingappa Pallagatti, Balwinder Singh, Nidhi Puri, Ravinder Singh, Aman Kalucha. Sonoporation, a redefined ultrasound modality as therapeutic aid: A review. J Clin Exp Dent 2011; 3(3):228234.

7. K wangjae Cho, Xu Wang, Shuming Nie, et al. Therapeutic nanoparticles for drug delivary. Clin Cancer Res 2008; 14(5):1315.

8. Nakashima M, Mizunuma $\mathrm{K}$,Murakami $\mathrm{T}$, Akamine $\mathrm{A}$. Induction of Reparative Dentin Formation by UItrasoundMediated Gene Delivery of Growth/Differentiation Factor. Human Gene Therapy 2003; 14:591-597.

9. Osawa K, Okubo Y, Nakao K, Koyama N, Bessho K.Osteoinduction. by microbubble-enhanced transcutaneous sonoporation of human bone morphogenetic protein-2. J of Gene Med 2009; 11(7): 633-641.

10. Mindaugas Tamosiunas, Rytis J urkonis, Lluis M. Mir, Arunas Lukosevicius, Mindaugas S. Venslauskas, Saulius
Satkauskas. Microbubble sonodestruction rate as a metric to evaluate sonoporation efficiency.J Ultrasound Med 2012; 31:1993-2000.

11. Suzuki R, Oda Y, Namai E,Takizawa T, N egishi Y, Utoguchi $\mathrm{N}$, Maruyama K. Development of site specific gene delivery system with sonoporation. Yakugaku Zasshi 2008; 128(2):187-192.

12. Liang $H$, Tang J , Halliwell M. Sonoporation, drug delivery. Gene therapy Proc Inst Mech Eng H. 2010; 224(2):343-361.

13. Sonoda S, Tachibana K, Uchino E et al. Epithelium and Keratocytes Mediated by UItrasound with Microbubbles. Invest Ophthalmol Vis Sci 2006; 47:558-564.

14. Lawrie A, Brisken AF, Francis SE. Gene expression after transfection of vascular cells in vitro. Circulation 1999; 99: 2617-2612.

15. Taniyama Y, Tachibana K, Hiraoka K, Namba T, Yamasaki $\mathrm{K}$, Hashiyav $\mathrm{N}$, et al. Local delivery of plasmid DNA into rat carotid artery v using ultrasound. Circulation 2002, 105: 1233-1239.

16. Kotopoulis S, Delalande A, Popa M, Mamaeva V, Dimcevski G, Gilja OH, Postema M, Gjertsen BT, McCormack E. Sonoporation-enhanced chemotherapy significantly reduces primary tumour burden in an orthotopic pancreatic cancer xenograft. Mol Imaging Biol 2014; 16(1):53-62.

17. Wang X, Lewis T, Mitchell D. The tumoricidal effect of sonodynamic therapy (SDT) on S -180 sarcoma in mice. Integr Cancer Ther 2008; 7: 96-102.

18. Korbelik M. PDT-associated host response and its role in the therapy outcome. Lasers Surg Med 2006; 38: 500-508.

19. Castano A P, Mroz P, H amblin MR. Photodynamic therapy and antitumour immunity. Nat Rev Cancer 2006; 6: 535545.

20. Korbelik M, Stott B, Sun J. Photodynamic therapygenerated vaccines: relevance of tumour cell death expression Brit J Cancer 2007; 97: 1381-1387.

\title{
Gain quick access to our journal online View our journal at
}

\author{
www.nacd.in
}

